\title{
Optimal Dispatch of Residential Photovoltaic Inverters Under Forecasting Uncertainties
}

\author{
Emiliano Dall'Anese, Member, IEEE, Sairaj V. Dhople, Member, IEEE, Brian B. Johnson, Member, IEEE, \\ and Georgios B. Giannakis, Fellow, IEEE
}

\begin{abstract}
Efforts to ensure reliable operation of existing lowvoltage distribution systems with high photovoltaic (PV) generation have focused on the possibility of inverters providing ancillary services such as active power curtailment and reactive power compensation. Major benefits include the possibility of averting overvoltages, which may otherwise be experienced when PV generation exceeds the demand. This paper deals with ancillary service procurement in the face of solar irradiance forecasting errors. In particular, assuming that the forecasted PV irradiance can be described by a random variable with known (empirical) distribution, the proposed uncertainty-aware optimal inverter dispatch (OID) framework indicates which inverters should provide ancillary services with a guaranteed a-priori risk level of PV generation surplus. To capture forecasting errors, and strike a balance between risk of overvoltages and (re)active power reserves, the concept of conditional value-at-risk is advocated. Due to $\mathrm{AC}$ power balance equations and binary inverter selection variables, the formulated OID involves the solution of a nonconvex mixed-integer nonlinear program. However, a computationally-affordable convex relaxation is derived by leveraging sparsity-promoting regularization approaches and semidefinite relaxation techniques.
\end{abstract}

Index Terms-Distribution networks, microgrids, photovoltaic systems, inverter control, optimal power flow, forecasting errors, conditional value-at-risk, voltage regulation.

\section{INTRODUCTION}

Deployment of photovoltaic (PV) systems in residential settings promises a multitude of environmental and economic advantages, including a sustainable capacity expansion of distribution systems. However, a unique set of challenges related to power quality, efficiency, and reliability may emerge, especially when an increased number of PV systems are deployed in existing distribution networks, and operate according to current practices [1], [2]. One challenge is associated with overvoltages when PV generation exceeds demand [3].

To ensure reliable operation of existing distribution feeders even during peak PV generation hours, recent efforts have

Paper submitted on June 29, 2014; revised September 21, 2014; accepted October 16, 2014. This work was supported by the Institute of Renewable Energy and the Environment (IREE) grant no. RL-0010-13, University of Minnesota, by the Laboratory Directed Research and Development (LDRD) Program at the National Renewable Energy Laboratory, and by the National Science Foundation (NSF) grants CCF 1423316 and CyberSEES 1442686.

E. Dall'Anese, S. V. Dhople, and G. B. Giannakis are with the Department of Electrical and Computer Engineering, and also the Digital Technology Center, University of Minnesota, 200 Union Street SE, Minneapolis, MN, USA; e-mail: $\{$ emiliano, sdhople, georgios\}eumn.edu. B. B. Johnson is with the National Renewable Energy Laboratory, Golden, CO, USA; e-mail: brian. johnsonenrel.gov. focused on the possibility of inverters providing ancillary services [4]-[6]. For instance, reactive power compensation approaches have been recognized as a viable option to effect voltage regulation at the medium-voltage distribution level [7]-[9]. The amount of reactive power injected or absorbed by inverters can be computed based on either local droop-type proportional laws [7], [8] or optimal power flow (OPF) strategies [9]. Either way, voltage regulation with this approach comes at the expense of low power factors at the substation and high network currents, with the latter leading to high power losses in the network [3]. Alternative approaches rely on operating inverters at unity power factor while curtailing part of the available active power [3], [10]. Active power curtailment strategies are particularly effective in the low-voltage portion of distribution feeders, where the high resistance-to-inductance ratio of low-voltage overhead lines renders voltage magnitudes more sensitive to variations in the active power injections. An optimal inverter dispatch (OID) framework was proposed in [11] to set both active and reactive power setpoints so that the network operation is optimized according to chosen criteria (e.g., minimizing power losses), while ensuring voltage regulation and adhering to other electrical constraints.

The approaches in [3], [7]-[12] are suitable for real-time network control, where the setpoints of the inverters scheduled to provide ancillary services are fine-tuned based on instantaneous load measurements and prevailing ambient conditions. Distinct from [3], [7]-[12], the problem of ancillary service procurement is considered in this paper. Specifically, ancillary service procurement refers here to the task of scheduling the inverters that will be required to provide ancillary services (in e.g., minute-, hour- or day-ahead markets [5], [6]), as well as quantifying both reactive reserves of the selected inverters and the active powers that inverters may be required to curtail. In this case, system operators cannot solely rely on the expected irradiance conditions to quantify the amount of ancillary services to provision, and irradiance forecasting errors must be taken into account. In fact, an excess of generation (compared to the expected one) may require additional inverters other than the ones scheduled (without accounting for forecasting errors) to provide ancillary services in order to avoid overvoltages.

The OID framework recently proposed in [11] is considerably broadened here by leveraging tools from risk-aware portfolio optimization to account for solar irradiance forecasting errors. Distinct from the real-time optimization method in [11], the approach developed in this paper enables effective provisioning of ancillary services in minute-, hour-, and dayahead markets [5], [6], by identifying the subset of critical 
PV inverters that will strongly impact both voltages and network performance objectives, and quantifying the amount of ancillary services that should be secured from each of the selected PV inverters. Specifically, for a given distribution of forecasting errors, the novel uncertainty-aware OID returns the amount of active power that can be curtailed in order to ensure voltage regulation with arbitrarily high probability and the amount of reactive power necessary to fulfill additional objectives. The proposed scheme is grounded on an AC power flow model, and it involves the solution of an OPF type problem encapsulating well-defined performance criteria and operational constraints. To capture forecasting errors, the conditional value-at-risk (CVaR) is advocated [13], [14], and utilized to trade off risks of overvoltage conditions for active power curtailment and reactive power compensation capabilities. Further, the resultant uncertainty-aware OID scheme involves the solution of a convex program and handles arbitrary probability distributions for the forecasting errors [15], [16].

Related prior works include, e.g., [17], where chanceconstrained optimal power flow (OPF) approaches were considered for high-voltage transmission systems with uncertain wind generation; a DC power flow approximation was utilized, along with Gaussian-distributed wind forecasting errors. Multi-period DC OPF was considered in e.g., [18], [19], where generation uncertainty was accounted for, while computing the schedule for controllable devices that minimize the expected operating costs. Upper bounds on the chance constraints based on e.g., Markov and Chebyshev inequalities were explored in [19]. Finally, extensions to the unit commitment problem can be found in [20]. At the distribution level, a chanceconstrained DC OPF formulation was developed in [21] to mitigate the effects of Gaussian-distributed forecasting errors on line currents and voltages. However, the DC power flow approximation may not be suitable for low-voltage resistive networks. Additional distributions for the renewable generation were considered in [22], where a nonconvex chanceconstrained AC OPF was formulated, and solved via offthe-shelf routines for nonlinear (nonconvex) programs. An economic dispatch problem in the presence of uncertain wind generation was proposed in [23]; in lieu of chance constraints, the cost of the problem was regularized with CVaR-type terms capturing the risk of generation shortage.

The remainder of the paper is organized as follows. System modeling is outlined in Section II] along with an overview of the OID with perfect knowledge of solar irradiance [11]. Basics of CVaR are provided in Section III-A whereas the uncertainty-aware-OID is outlined in Section III-B Case studies are discussed in IV while Section $\nabla$ concludes the paper 1

\footnotetext{
${ }^{1}$ Notation: Upper-case (lower-case) boldface letters will be used for matrices (column vectors); $(\cdot)^{\top}$ for transposition; $(\cdot)^{*}$ complex-conjugate; and, $(\cdot)^{\mathrm{H}}$ complex-conjugate transposition; $\Re\{\cdot\}$ and $\Im\{\cdot\}$ denote the real and imaginary parts of a complex number, respectively; $\mathrm{j}:=\sqrt{-1}$ the imaginary unit. $\mathbb{R}_{+}:=\{x \in \mathbb{R}: x \geq 0\} ; \operatorname{Tr}(\cdot)$ the matrix trace; $\operatorname{rank}(\cdot)$ the matrix rank; $|\cdot|$ denotes the magnitude of a number or the cardinality of a set; $\|\mathbf{v}\|_{2}:=\sqrt{\mathbf{v}^{\mathrm{H}} \mathbf{v}}$; and $\|\cdot\|_{F}$ stands for the Frobenius norm. For any $x \in \mathbb{R}$, $[x]_{+}:=\max \{0, x\} . \mathbb{I}_{\{A\}}$ is the indicator function (i.e., $\mathbb{I}_{\{A\}}=1$ if event $A$ is true, and 0 otherwise). Finally, $\mathbf{I}_{N}$ denotes the $N \times N$ identity matrix; and, $\mathbf{0}_{M}, \mathbf{1}_{M}$ the $M \times 1$ vectors with all zeroes and ones, respectively.
}

\section{Preliminaries}

\section{A. Network and inverter models}

Consider a distribution system comprising $N+1$ nodes collected in the set $\mathcal{N}:=\{0,1, \ldots, N\}$ (node 0 denotes the secondary of the step-down transformer), and lines represented by the set of edges $\mathcal{E}:=\{(m, n)\} \subset \mathcal{N} \times \mathcal{N}$. Subsets $\mathcal{U}, \mathcal{H} \subset \mathcal{N}$ collect nodes corresponding to utility poles and households with installed PV inverters, respectively. For simplicity of exposition, a balanced system is considered; however, the framework proposed subsequently can be extended to unbalanced multi-phase systems following the method in [24].

Let $V_{n} \in \mathbb{C}$ and $I_{n} \in \mathbb{C}$ denote the phasors for the lineto-ground voltage and the current injected at node $n \in \mathcal{N}$, respectively, and define $\mathbf{i}:=\left[I_{0}, I_{1}, \ldots, I_{N}\right]^{\top} \in \mathbb{C}^{N+1}$ and $\mathbf{v}:=\left[V_{0}, V_{1}, \ldots, V_{N}\right]^{\top} \in \mathbb{C}^{N+1}$. Using Ohm's and Kirchhoff's circuit laws, the linear relationship $\mathbf{i}=\mathbf{Y v}$ can be established, where the system admittance matrix $\mathbf{Y} \in \mathbb{C}^{N+1 \times N+1}$ is formed based on the system topology and the $\pi$-equivalent circuits of the lines $(m, n) \in \mathcal{E}$; see e.g., [11], [24], [25]. A constant $P Q$ model [26] is adopted for the load, with $P_{\ell, h}$ and $Q_{\ell, h}$ denoting the active and reactive demands at node $h \in \mathcal{H}$, respectively (clearly, $P_{\ell, h}=Q_{\ell, h}=0$ for all $h \in \mathcal{U}$ ).

For given solar irradiation conditions, let $P_{h}^{\text {av }}$ denote the available active power from the PV array at node $h \in \mathcal{H}$. Following business-as-usual practices [2], grid-tied inverters operate at the unity-power-factor setpoint $\left(P_{h}^{\text {av }}, 0\right)$. To address emerging overvoltage and power quality concerns [1], inverters may be called upon to provide ancillary services [4], [5]. These include e.g., Volt/VAR support [7]-[9] and active power curtailment [3], with the allowed inverter operating regime on the complex-power plane illustrated in Fig. 11a) and 1(b), respectively. The OID framework in [11] offers increased flexibility over Volt/VAR support and active power curtailment, by invoking a joint control of real and reactive powers produced by PV inverters. In particular, the allowed operating regime for the PV inverter at household $h$ is illustrated in Fig. 11d) and described by

$$
\mathcal{F}_{h}^{\text {OID }}\left(P_{h}^{\mathrm{av}}\right):=\left\{\begin{array}{c}
0 \leq P_{c, h} \leq P_{h}^{\mathrm{av}} \\
P_{c, h}, Q_{c, h}: Q_{c, h}^{2} \leq S_{h}^{2}-\left(P_{h}^{\mathrm{av}}-P_{c, h}\right)^{2} \\
\left|Q_{c, h}\right| \leq \tan \theta\left(P_{h}^{\mathrm{av}}-P_{c, h}\right)
\end{array}\right\}
$$

where $P_{c, h}$ is the active power curtailed, $Q_{c, h}$ is the reactive power injected $\left(Q_{c, h}>0\right)$ or absorbed $\left(Q_{c, h}<0\right)$, and $S_{h}$ is the apparent power rating. In the absence of minimum power factor constraints (i.e., $\theta=\pi / 2$ ), the operating region corresponds to the one in Fig. 1(c).

\section{B. Optimal inverter dispatch with known available powers}

An overview of the OID with perfect knowledge of $\left\{P_{h}^{\text {av }}\right\}_{h \in \mathcal{H}}$ is provided next, to lay the foundation for the uncertainty-aware framework outlined in Section III

For given available powers $\left\{P_{h}^{\text {av }}\right\}_{h \in \mathcal{H}}$, the objective of the OID is to identify the critical inverters that should be dispatched in order to ensure electrical network constraints, and compute their optimal steady-state active/reactive power setpoints. To this end, let $z_{h}$ be a binary optimization variable indicating whether PV inverter $h$ provides ancillary services 


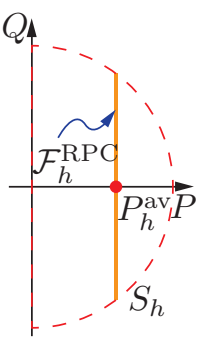

(a)

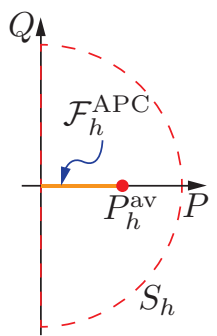

(b)

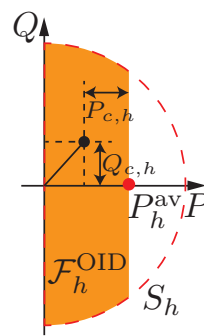

(c)

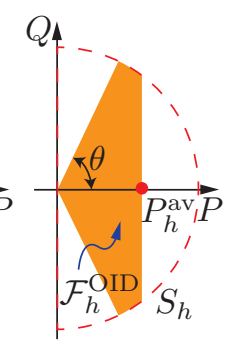

(d)
Fig. 1. Feasible operating regions for the $h$-th inverter with apparent power rating $S_{h}$ under a) reactive power control, b) active power curtailment, c) OID with joint control of real and reactive power, and d) OID with a lower-bound on power factor [11].

$\left(z_{h}=1\right)$ or not $\left(z_{h}=0\right)$, and let $\mathbf{p}_{c} \in \mathbb{R}_{+}^{|\mathcal{H}|}, \mathbf{q}_{c} \in \mathbb{R}^{|\mathcal{H}|}$ be vectors collecting the active powers curtailed and the reactive powers injected/absorbed by the inverters. Further, let $C\left(\mathbf{v}, \mathbf{p}_{c}\right)$ be a given cost function capturing network- and customer-oriented objectives [11]; for instance, $C\left(\mathbf{v}, \mathbf{p}_{c}\right)$ may account for active power losses in the network and possible costs associated with active power set points [5]. With these definitions, a rendition of the OID task is formulated as:

$$
\begin{aligned}
& \min _{\mathbf{v}, \mathbf{i}, \mathbf{p}_{c}, \mathbf{q}_{c},\left\{z_{h}\right\}} C\left(\mathbf{v}, \mathbf{p}_{c}\right)+c_{z} \sum_{h \in \mathcal{H}} z_{h} \\
& \text { subject to } \mathbf{i}=\mathbf{Y v},\left\{z_{h}\right\} \in\{0,1\}^{|\mathcal{H}|}, \text { and } \\
& V_{h} I_{h}^{*}=\left(P_{h}^{\text {av }}-P_{c, h}-P_{\ell, h}\right)+\mathrm{j}\left(Q_{c, h}-Q_{\ell, h}\right) \\
& V_{n} I_{n}^{*}=0 \quad \forall n \in \mathcal{U} \\
& V^{\text {min }} \leq\left|V_{n}\right| \leq V^{\max } \forall n \in \mathcal{N} \\
&\left(P_{c, h}, Q_{c, h}\right) \in\left\{\begin{array}{ll}
\{(0,0)\}, & \text { if } z_{h}=0 \\
\mathcal{F}_{h}^{\text {OID }}, & \text { if } z_{h}=1
\end{array} \quad \forall h \in \mathcal{H}\right.
\end{aligned}
$$

where the balance constraint $1 \mathrm{lb}$ is enforced at each node $h \in \mathcal{H}$; 1e indicates which inverters have to be dispatched (i.e., $\left(P_{c, h}, Q_{c, h}\right) \in \mathcal{F}_{h}^{\text {OID }}$ ), or, operate in the business-asusual mode (i.e., $\left.\left(P_{c, h}, Q_{c, h}\right)=(0,0)\right)$; and, the constraint on $V_{0}$ is left implicit. Finally, $c_{z} \in \mathbb{R}_{+}$is a weighting coefficient, used to trade off achievable cost $C\left(\mathbf{v}, \mathbf{p}_{c}\right)$ for the number of controlled inverters. When $c_{z}$ represents a fixed reward for customers providing ancillary services [5] and $C\left(\mathbf{v}, \mathbf{p}_{c}\right)$ models costs associated with active power losses and active power set points, OID (11) returns the inverter setpoints that minimize the economic cost incurred by the feeder operation.

Unfortunately, problem (1) is nonconvex and it contains binary variables; thus, it is challenging to solve optimally and efficiently, even by utilizing off-the-shelf solvers for mixedinteger nonlinear programs. Nevertheless, a computationallyaffordable convex reformulation was introduced in [11], by leveraging contemporary sparsity-promoting regularization [27] and semidefinite relaxation (SDR) techniques [24], [25] as summarized next.

To address the non-convexity of constraints (1b)- $1 \mathrm{dd}$, consider expressing powers and voltage magnitudes as linear functions of the outer-product complex Hermitian matrix $\mathbf{V}:=\mathbf{v v}^{\mathrm{H}}$, and to reformulate the OID problem with cost and constraints that are linear functions of $\mathbf{V}$. Specifically, define the matrix $\mathbf{Y}_{n}:=\mathbf{e}_{n} \mathbf{e}_{n}^{\top} \mathbf{Y}$ per node $n$, where $\left\{\mathbf{e}_{n}\right\}_{n \in \mathcal{N}}$ denotes the canonical basis of $\mathbb{R}^{|\mathcal{N}|}$. Further, based on $\mathbf{Y}_{n}$, define also the Hermitian matrices $\mathbf{A}_{n}:=\frac{1}{2}\left(\mathbf{Y}_{n}+\mathbf{Y}_{n}^{\mathbf{H}}\right), \mathbf{B}_{n}:=$
$\frac{j}{2}\left(\mathbf{Y}_{n}-\mathbf{Y}_{n}^{\mathrm{H}}\right)$, and $\mathbf{M}_{n}:=\mathbf{e}_{n} \mathbf{e}_{n}^{\top}$. Then, the node balance constraints for active and reactive powers can be equivalently expressed as $\operatorname{Tr}\left(\mathbf{A}_{h} \mathbf{V}\right)=P_{h}^{\text {av }}-P_{c, h}-P_{\ell, h}$ and $\operatorname{Tr}\left(\mathbf{B}_{h} \mathbf{V}\right)=$ $Q_{c, h}-Q_{\ell, h}$, respectively. Similarly, constraint (1d) can be equivalently expressed as $V_{\min }^{2} \leq \operatorname{Tr}\left(\mathbf{M}_{n} \mathbf{V}\right) \leq V_{\max }^{2}$. The technical constraints $\mathbf{V} \succeq \mathbf{0}$ and $\operatorname{rank}(\mathbf{V})=1$ need to be added, to ensure recoverability of the voltage vector $\mathbf{v}$ [24], [25]. The only source of non-nonconvexity is now constraint $\operatorname{rank}(\mathbf{V})=1$; however in the spirit of SDR, this constraint can be dropped. If the optimal solution of the relaxed problem has rank 1 , then the resultant power flows are globally optimal for given inverter setpoints.

As for the binary variables $\left\{z_{h}\right\}$, notice first that if PV inverter $h$ is not selected for ancillary services, one has that $P_{c, h}=Q_{c, h}=0$ [cf. (1e)]. Thus, assuming that only a subset of PV inverters may need to be controlled in order to ensure electrical network constraints and minimize 1 a , one has that the $2|\mathcal{H}| \times 1$ real-valued vector $\left[\mathbf{p}_{c}^{\top}, \mathbf{q}_{c}^{\top}\right]^{\top}$ is group sparse [27]; that is, either the $2 \times 1$ sub-vectors $\left[P_{c, h}, Q_{c, h}\right]^{\top}$ equal to $\mathbf{0}$, or not. In lieu of binary variables, this group-sparsity attribute enables PV inverter selection by regularizing the cost in (1) with the following group-sparsity-promoting function:

$$
G\left(\mathbf{p}_{c}, \mathbf{q}_{c}\right):=c_{z} \sum_{h \in \mathcal{H}}\left\|\left[P_{c, h}, Q_{c, h}\right]\right\|_{2} .
$$

Leveraging these tools, a relaxation of the OID problem is obtained as:

$$
\begin{array}{ll}
\min _{\mathbf{V}, \mathbf{p}_{c}, \mathbf{q}_{c}} C\left(\mathbf{V}, \mathbf{p}_{c}\right)+G\left(\mathbf{p}_{c}, \mathbf{q}_{c}\right) & \\
\text { s. to } \mathbf{V} \succeq \mathbf{0}, \text { and } & \\
\operatorname{Tr}\left(\mathbf{A}_{h} \mathbf{V}\right)=-P_{\ell, h}+P_{h}^{\text {av }}-P_{c, h} & \forall h \in \mathcal{H} \\
\operatorname{Tr}\left(\mathbf{B}_{h} \mathbf{V}\right)=-Q_{\ell, h}+Q_{c, h} & \forall h \in \mathcal{H} \\
\operatorname{Tr}\left(\mathbf{A}_{n} \mathbf{V}\right)=0, \operatorname{Tr}\left(\mathbf{B}_{n} \mathbf{V}\right)=0 & \forall n \in \mathcal{U} \\
V_{\min }^{2} \leq \operatorname{Tr}\left(\mathbf{M}_{n} \mathbf{V}\right) \leq V_{\max }^{2} & \forall n \in \mathcal{N} \\
\quad\left(P_{c, h}, Q_{c, h}\right) \in \mathcal{F}_{h}^{\text {OII }}\left(P_{h}^{\text {av }}\right) & \forall h \in \mathcal{H} .
\end{array}
$$

Problem (3) is convex, and can be readily re-formulated in a standard semidefinite programming (SDP) form by resorting to the epigraph forms of $G\left(\mathbf{p}_{c}, \mathbf{q}_{c}\right)$ and $C\left(\mathbf{V}, \mathbf{p}_{c}\right)$ [28], as well as the linear matrix inequality form of $Q_{c, h}^{2} \leq S_{h}^{2}-\left(P_{h}^{\text {av }}-P_{c, h}\right)^{2}$ obtainable by using the Shur complement.

When the distribution system is balanced and radial, sufficient conditions for obtaining a rank-1 solution in SDRbased OPF-type reformulations are available in [29], [30], and they can be conveniently tailored to (3); for example, one requirement is that the cost $3 \mathrm{ab}$ is increasing in the injected active powers. What is more, constraint $\mathbf{V} \succeq \mathbf{0}$ can be equivalently re-written as $\mathbf{V}^{(i, j)} \succeq \mathbf{0}, \forall(i, j) \in \mathcal{E}$, with $\mathbf{V}^{(i, j)}$ denoting the $2 \times 2$ sub-matrix of $\mathbf{V}$ corresponding to nodes $i$ and $j$. Since $\left|V_{n}\right|>0$ for all nodes, one has that each constraint $\mathbf{V}^{(i, j)} \succeq \mathbf{0}$ can be further re-expressed as $\left(\mathbf{V}_{i j}\right.$ is the $(i, j)$-th entry of $\mathbf{V})$

$$
\mathbf{V}_{i i}>0, \mathbf{V}_{j j}>0, \mathbf{V}_{i j}=\mathbf{V}_{j i}^{*} \text {, and }\left|\mathbf{V}_{i j}\right|^{2}-\mathbf{V}_{i i} \mathbf{V}_{j j} \leq 0 \text {, }
$$

which is a second-order cone constraint. Thus, for radial and balanced topologies, (3) can be transformed into a 
second-order cone program, with due computational advantages. The worst-case complexity of an SDP is on the or$\operatorname{der} \mathcal{O}\left(N_{v}^{4.5} \log (1 / \epsilon)\right)$ for general-purpose solvers, with $N_{v}$ denoting the total number of variables in the problem, and $\epsilon>0$ a given solution accuracy [28], [31]. The worstcase complexity of a second-order cone program is on the order of $\mathcal{O}\left(N_{v}^{3} \log (1 / \epsilon)\right)$ [31]. Notice however that sparsity in $\left\{\mathbf{A}_{n}, \mathbf{B}_{n}, \mathbf{M}_{n}\right\}$ and the chordal structure of the underlying electrical graph can be exploited to devise customized solvers with reduced computational burden; see e.g., [32]. Finally, extensions of (3) to multi-phase unbalanced distribution systems can be derived by following the method in [24].

Remark (ZIP load model). A constant power load model is utilized in the OID (11). However, the OID formulation can be broadened to account for constant-impedance, constantcurrent, and constant-power load components (i.e., the socalled ZIP model [33]) by following the method in [34]. For constant impedance loads, the demands are proportional to the voltage magnitude squared; thus, they can be easily incorporated in (3). On the other hand, since constant current loads are functions of the voltage magnitudes, appropriate reformulations of (3) are required. In particular, [34] suggests to replace matrix $\mathbf{V}$ with $\tilde{\mathbf{V}}:=\tilde{\mathbf{v}} \tilde{\mathbf{V}}^{\mathrm{H}}$, where the voltagerelated vector $\tilde{\mathbf{v}}$ is defined as $\tilde{\mathbf{v}}:=\left[1, \mathbf{v}^{\top}\right]^{\top}$ (clearly, matrices $\mathbf{A}_{n}, \mathbf{B}_{n}$, and $\mathbf{M}_{n}$ are re-defined accordingly). In this case, it may not be possible to find a rank-1 matrix $\tilde{\mathbf{V}}$, although the obtained solution yields a reasonably good approximation of the constant current loads; for more details, see the discussion provided in [34].

\section{InVERTER DisPatch Under ForeCASTING ERRORS}

For given available powers $\left\{P_{h}^{\text {av }}\right\}_{h \in \mathcal{H}}$, the OID task (3) identifies the inverters that must provide ancillary services in order to avoid overvoltage conditions [cf. [3e] ], and computes the steady-state setpoints that minimize the selected operational and economic objectives [cf. (3a)]. This approach is suitable for real-time network operation, based on instantaneous measurements of loads and available powers. On the other hand, solar irradiance forecasting errors must be taken into account when OID is utilized for ancillary-services procurement, in either day-ahead or hour-ahead ancillary service markets [5], [6]. In this case, system operators cannot rely on the expected value of the active power available from the PV array to quantify the amount of ancillary services to provision. In fact, an excess of generation (compared to the expected one) may require additional inverters other than the ones scheduled without accounting for forecasting errors, to deviate from the business-as-usual setpoint [1], [2].

In the remainder of this section, the so-called CVaR will be utilized to capture the risk of excess in the active power generation, and subsequently proactively select the inverters that will be required to provide ancillary services.

\section{A. Overview of the Conditional Value-at-Risk Approach}

An overview of the value-at-risk (VaR) and CVaR measures typically considered in risk-aware portfolio optimization [14] - is given in this subsection. These tools

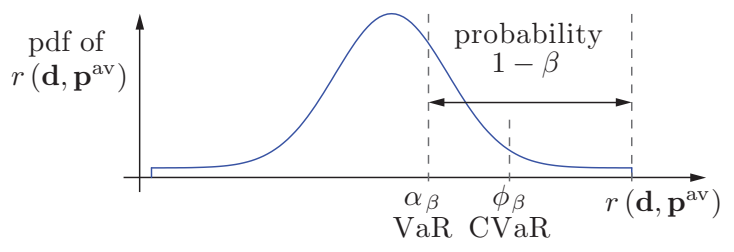

Fig. 2. Illustrative example of the CVaR associated with function $r\left(\mathbf{d}, \mathbf{p}^{\mathrm{av}}\right)$.

will be utilized to formulate the uncertainty-aware OID in Section III-B

Suppose $\mathbf{p}^{\text {av }}:=\left[P_{1}^{\mathrm{av}}, \ldots, P_{|\mathcal{H}|}^{\mathrm{av}}\right]^{\top}$ is a real-valued random vector, and let $\rho\left(\mathbf{p}^{\text {av }}\right)$ denote its probability density function. Assume that $\rho\left(\mathbf{p}^{\mathrm{av}}\right)$ is known (or an empirical estimate is available [15], [16]), and supported on a closed and bounded set $\mathcal{D} \subset \mathbb{R}^{|\mathcal{H}|}$. For example, in the context of solar irradiance forecasting, a viable choice for $\rho\left(\mathbf{p}^{\text {av }}\right)$ would be a truncated multivariate Gaussian distribution, as described in [15]. See also e.g., [16] for additional models for $\rho\left(\mathbf{p}^{\text {av }}\right)$ in the context of solar irradiance forecasting.

Let $r: \mathbb{R}^{|\mathcal{H}|} \times \mathcal{D} \rightarrow \mathbb{R}$ be a real-valued function of both the random vector $\mathbf{p}^{\text {av }}$ and the vector of presumed powers $\mathbf{d} \in \mathbb{R}^{|\mathcal{H}|}$. In particular, let $r\left(\mathbf{d}, \mathbf{p}^{\text {av }}\right)=\sum_{h \in \mathcal{H}}\left[P_{h}^{\text {av }}-d_{h}\right]_{+}$ capture possible excess of power generation during hours with high and yet uncertain generation (and, hence, the risk of overvoltages throughout the distribution feeder) 2 Henceforth, $r\left(\mathbf{d}, \mathbf{p}^{\text {av }}\right)$ will be referred to as the surplus generation function. Notice that $r\left(\mathbf{d}, \mathbf{p}^{\text {av }}\right)$ takes positive values only when $P_{h}^{\text {av }}>d_{h}$ for at least one inverter. For a given vector $\mathbf{d}, r\left(\mathbf{d}, \mathbf{p}^{\text {av }}\right)$ is a random variable with cumulative distribution function

$\Psi_{r}(\mathbf{d}, \alpha):=\operatorname{Pr}\left\{r\left(\mathbf{d}, \mathbf{p}^{\mathrm{av}}\right) \leq \alpha\right\}=\int_{\mathcal{D}} \mathbb{I}_{\{r(\mathbf{d}, \mathbf{p}) \leq \alpha\}} \rho(\mathbf{p}) \mathrm{d} \mathbf{p}$.

Notice that $\Psi_{r}(\mathbf{d}, \alpha)$ is continuous from the right (but not necessarily from the left), nondecreasing in $\alpha$, and parameterized by $\mathbf{d}$ [14]. Intuitively, $\Psi_{r}(\mathbf{d}, \alpha)$ quantifies the probability of the actual available power exceeding the presumed value $\mathbf{d}$. Based on (4), the VaR and CVaR measures are defined next (see [13], [14] for additional details).

For a user-prescribed probability level $\beta \in(0,1)$, the corresponding VaR, denoted as $\alpha_{\beta}$, associated with the random surplus generation function $r\left(\mathbf{d}, \mathbf{p}^{\text {av }}\right)$, is the left endpoint of the non-empty interval collecting the values of $\alpha$ for which $\Psi_{r}(\mathbf{d}, \alpha)=\beta$; i.e.,

$$
\alpha_{\beta}(\mathbf{d}):=\inf \left\{\alpha \in \mathbb{R}: \Psi_{r}(\mathbf{d}, \alpha) \geq \beta\right\} .
$$

For any $\mathbf{d}$, the $\mathrm{CVaR}$, denoted as $\phi_{\beta}(\mathbf{d})$, is the expected value of the surplus generation function when considering entries that are greater than or equal to $\alpha_{\beta}(\mathbf{d})$ :

$$
\phi_{\beta}(\mathbf{d}):=\frac{1}{1-\beta} \int_{\mathcal{D}} \mathbb{I}_{\left\{r(\mathbf{d}, \mathbf{p}) \geq \alpha_{\beta}(\mathbf{d})\right\}} r(\mathbf{d}, \mathbf{p}) \rho(\mathbf{p}) \mathrm{d} \mathbf{p} .
$$

In other words, in the $(1-\beta)$ percent of cases where $r\left(\mathbf{d}, \mathbf{p}^{\mathrm{av}}\right)=\sum_{h \in \mathcal{H}}\left[P_{h}^{\mathrm{av}}-d_{h}\right]_{+} \geq \alpha_{\beta}(\mathbf{d})$, the $\operatorname{CVaR} \phi_{\beta}(\mathbf{d})$

\footnotetext{
${ }^{2}$ Another viable choice is $r\left(\mathbf{d}, \mathbf{p}^{\mathrm{av}}\right)=\left[\sum_{h \in \mathcal{H}}\left(P_{h}^{\mathrm{av}}-d_{h}\right)\right]_{+}$; that is, the network-wide surplus of active power. However, $r\left(\mathbf{d}, \mathbf{p}^{\text {av }}\right)=\sum_{h \in \mathcal{H}}\left[P_{h}^{\text {av }}-\right.$ $\left.d_{h}\right]_{+}$captures local (as opposed to network-wide) random changes in the active power injections, and it is therefore a more suitable indicator for the risk of high active power flows in sections of the feeder.
} 
quantifies the expected amount of available active power further exceeding $\alpha_{\beta}(\mathbf{d})$. An illustrative example of the VaR and $\mathrm{CVaR}$ associated with a random function $r\left(\mathbf{d}, \mathbf{p}^{\text {av }}\right)$ is provided in Fig. 2, in this example, the probability density function of $r\left(\mathbf{d}, \mathbf{p}^{\text {av }}\right)$ is a truncated Gaussian. The CVaR is typically preferred over $\mathrm{VaR}$ as a risk measure, since it is coherent (in fact, VaR violates sub-additivity-one of the properties of a coherent measure [13]).

Key to utilizing CVaR as a performance objective in riskaware optimization tasks is the link established in [14] between $\phi_{\beta}(\mathbf{d})$ and the following real-valued function:

$$
R_{\beta}(\alpha, \mathbf{d}):=\alpha+\frac{1}{(1-\beta)} \int_{\mathcal{D}}[r(\mathbf{d}, \mathbf{p})-\alpha]_{+} \rho(\mathbf{p}) \mathrm{d} \mathbf{p} .
$$

Specifically, [14, Thm. 1] asserts the following three facts:

(F1) $R_{\beta}(\alpha, \mathbf{d})$ is convex and continuously differentiable in $\alpha$. (F2) For any $\mathbf{d} \in \mathbb{R}^{|\mathcal{H}|}$, the CVaR $\phi_{\beta}(\mathbf{d})$ represents the minimum value of $R_{\beta}(\alpha, \mathbf{d})$; that is,

$$
\phi_{\beta}(\mathbf{d})=\min _{\alpha \in \mathbb{R}} R_{\beta}(\alpha, \mathbf{d}) .
$$

(F3) The set of minimizers

$$
\mathcal{A}_{\beta}(\mathbf{d}):=\arg \min _{\alpha \in \mathbb{R}} R_{\beta}(\alpha, \mathbf{d})
$$

is closed and bounded, and the $\operatorname{VaR} \alpha_{\beta}(\mathbf{d})$ is the left endpoint of this interval.

An advantage of the integral function (7) is that an empirical estimate of $R_{\beta}(\alpha, \mathbf{d})$ can be obtained via sample averaging. This is especially useful in cases when the integral in (7) cannot be evaluated in closed-form. For instance, given $S$ Monte Carlo samples, $\left\{\mathbf{p}^{\text {av }}[s] \in \mathcal{D}\right\}_{s=1}^{S}$, of the random vector $\mathbf{p}^{\text {av }}$, a distribution-free approximation of $R_{\beta}(\alpha, \mathbf{d})$ is given by

$$
\hat{R}_{\beta}(\alpha, \mathbf{d})=\alpha+\frac{1}{S(1-\beta)} \sum_{s=1}^{S}\left[r\left(\mathbf{d}, \mathbf{p}^{\mathrm{av}}[s]\right)-\alpha\right]_{+},
$$

and, for a sufficiently high number of samples $S$, almost sure convergence of $\hat{R}_{\beta}(\alpha, \mathbf{d})$ to $R_{\beta}(\alpha, \mathbf{d})$ is guaranteed by the (strong) law of large numbers. Compared to (7), the sample average $\hat{R}_{\beta}(\alpha, \mathbf{d})$ is not differentiable due to the projection operator $[\cdot]_{+}$. However, this hurdle can be easily overcome by resorting to the epigraph form of $\hat{R}_{\beta}(\alpha, \mathbf{d})$ [35].

To consider $\hat{R}_{\beta}(\alpha, \mathbf{d})$ (or, its epigraph form) in multiobjective optimization problems, convexity with respect to (wrt) both $\alpha$ and $\mathbf{d}$ is desirable. To this end, the following claims established in [14, Thm. 2] can be conveniently leveraged:

(C1) If function $r(\mathbf{d}, \mathbf{p})$ is convex in $\mathbf{d}$, then $R_{\beta}(\alpha, \mathbf{d})$ is jointly convex in $\mathbf{d}$ and $\alpha$; and $\phi_{\beta}(\mathbf{d})$ is convex in $\mathbf{d}$.

(C2) The following equality holds

$$
\min _{\mathbf{d} \in \mathbb{R}^{|\mathcal{H}|}} \phi_{\beta}(\mathbf{d})=\min _{\mathbf{d} \in \mathbb{R}^{|\mathcal{H}|}, \alpha \in \mathbb{R}} R_{\beta}(\alpha, \mathbf{d}),
$$

and $\mathbf{d}^{\star}, \alpha^{\star}$ are minimizers of $R_{\beta}(\alpha, \mathbf{d})$ if and only if $\mathbf{d}^{\star}$ is a minimizer of $\phi_{\beta}(\mathbf{d})$ and $\alpha^{\star} \in \mathcal{A}_{\beta}\left(\mathbf{d}^{\star}\right)$.

Claim (C2) asserts that minimizing the CVaR wrt to the variables $\mathbf{d}$ is equivalent to jointly minimizing $R_{\beta}(\alpha, \mathbf{d}$ ) (and thus $\hat{R}_{\beta}(\alpha, \mathbf{d})$ ) over $\mathbf{d}$ and $\alpha$, with the $\operatorname{VaR} \alpha^{\star}$ coming out as a byproduct. This feature will be exploited in the risk-aware OID framework outlined next, where $\mathbf{d}$ represents the vector of presumed available powers associated with a given CVaR.

\section{B. Risk-aware inverter dispatch}

For $r\left(\mathbf{d}, \mathbf{p}^{\text {av }}\right)=\sum_{h \in \mathcal{H}}\left[P_{h}^{\text {av }}-d_{h}\right]_{+}$, function $R_{\beta}(\alpha, \mathbf{d})$ is jointly convex in $\mathbf{d}$ and $\alpha$ by virtue of (C1). Further, given $S$ independent samples $\left\{\mathbf{p}^{\mathrm{av}}[s] \in \mathcal{D}\right\}_{s=1}^{S}$, an approximation of $R_{\beta}(\alpha, \mathrm{d})$ is given by [cf. [10]

$$
\hat{R}_{\beta}(\alpha, \mathbf{d})=\alpha+\frac{1}{S(1-\beta)} \sum_{s=1}^{S}\left[\sum_{h \in \mathcal{H}}\left[P_{h}^{\mathrm{av}}[s]-d_{h}\right]_{+}-\alpha\right]_{+} .
$$

Thus, given $\beta$ and the Monte Carlo samples $\left\{\mathbf{p}^{\mathrm{av}}[s] \in\right.$ $\mathcal{D}\}_{s=1}^{S}$, the objective of the risk-aware OID problem is to jointly minimize the OID objective (3a) under the presumed available power levels $\mathbf{d}$, as well as the risk of additional available power surplus, subject to the $\mathrm{AC}$ power flow and OID-related inverter constraints; that is,

$$
\begin{aligned}
& \min _{\mathbf{V}, \mathbf{p}_{c}, \mathbf{q}_{c}, \mathbf{d}, \alpha} C\left(\mathbf{V}, \mathbf{p}_{c}, \mathbf{d}\right)+G\left(\mathbf{p}_{c}, \mathbf{q}_{c}\right)+c_{R} \hat{R}_{\beta}(\alpha, \mathbf{d}) \\
& \text { s. to } \mathbf{V} \succeq \mathbf{0} \text {, and } \\
& \operatorname{Tr}\left(\mathbf{A}_{h} \mathbf{V}\right)=-P_{\ell, h}+d_{h}-P_{c, h} \forall h \in \mathcal{H} \\
& \operatorname{Tr}\left(\mathbf{B}_{h} \mathbf{V}\right)=-Q_{\ell, h}+Q_{c, h} \forall h \in \mathcal{H} \\
& \operatorname{Tr}\left(\mathbf{A}_{n} \mathbf{V}\right)=0, \operatorname{Tr}\left(\mathbf{B}_{n} \mathbf{V}\right)=0 \forall n \in \mathcal{U} \\
& V_{\min }^{2} \leq \operatorname{Tr}\left(\mathbf{M}_{n} \mathbf{V}\right) \leq V_{\max }^{2} \forall n \in \mathcal{N} \\
&\left(P_{c, h}, Q_{c, h}\right) \in \mathcal{F}_{h}^{\text {OID }}\left(d_{h}\right) \forall h \in \mathcal{H} \\
& \mathbf{d} \in \mathcal{D}
\end{aligned}
$$

where $c_{R} \in \mathbb{R}_{+}$is a predetermined parameter, used to trade off achievable CVaR values for OID objectives at the $\beta$-risk level. Problem (13) is convex, and can be re-stated in either standard SDP form by using the epigraph form of [13a [28], or, in standard SOCP form for systems that are radial and balanced.

To appreciate the usefulness of the CVaR risk measure, suppose that for given $\beta$, it turns out that at least $H<|\mathcal{H}|$ inverters are required to curtail at most $\left\{\bar{P}_{c, h}\right\} \mathrm{W}$, in order to ensure voltage regulation in the $\beta$ percent of the cases; that is, whenever $\sum_{h \in \mathcal{H}}\left[P_{h}^{\text {av }}-d_{h}\right]_{+} \leq \alpha_{\beta}(\mathbf{d})$. Then, minimizing the $\mathrm{CVaR} \phi_{\beta}(\mathbf{d})$ is equivalent to minimizing the additional amount of active powers that inverters may be required to curtail in case of unexpected over-generation (i.e., when $\left.\sum_{h \in \mathcal{H}}\left[P_{h}^{\mathrm{av}}-d_{h}\right]_{+} \geq \alpha_{\beta}(\mathbf{d})\right)$, or, minimizing the number of additional inverters that may be called upon to provide ancillary services. Elaborating further on the impacts of uncertainties on the system operational costs, suppose that function $C\left(\mathbf{V}, \mathbf{p}_{c}, \mathbf{d}\right)$ is set to $C\left(\mathbf{V}, \mathbf{p}_{c}, \mathbf{d}\right)=c_{L}\left(\mathbf{1}_{|\mathcal{H}|}^{\top}\left(\mathbf{d}-\mathbf{p}_{c}\right)+P_{0}\right)+$ $c_{P} \mathbf{1}_{|\mathcal{H}|}^{\top} \mathbf{p}_{c}$, where the first term captures the cost incurred by power losses in the network, the available powers are $\mathbf{d}$, and the second term models the cost of active power that can be curtailed; see e.g., [5]. Further, recall that $G\left(\mathbf{p}_{c}, \mathbf{q}_{c}\right)$ accounts for possible fixed rewards for customers when their inverters are called upon to provide ancillary reserves. If $c_{R}$ quantifies the economic loss incurred by overvoltages, then (13a) strikes a balance between system operational costs when operated at a risk level $\beta$, and the economic loss that the system may incur in case of unexpected generation surplus. Section IV will elaborate further on how to trade off CVaR for the amount of ancillary services to be provisioned. 
To re-state (13) in a standard SDP form (similar steps can be followed for the SOCP case), assume for simplicity that $C\left(\mathbf{V}, \mathbf{p}_{c}, \mathbf{d}\right)$ is linear in its arguments. Consider then introducing the non-negative auxiliary vector variable $\mathbf{z}:=$ $\left[z_{1}, \ldots, z_{|\mathcal{H}|}\right]^{\top}$, replace $G\left(\mathbf{p}_{c}, \mathbf{q}_{c}\right)$ with $c_{z} \mathbf{1}_{|\mathcal{H}|}^{\top} \mathbf{z}$ in $(13 \mathrm{a})$, and add constraints $\left\|\left[P_{c, h}, Q_{c, h}\right]\right\|_{2} \leq z_{h}$, for all $h \in \mathcal{H}$. Then, by introducing auxiliary variables $\mathbf{y} \in \mathbb{R}^{S}$ and $\left\{\mathbf{u}_{s} \in \mathbb{R}^{|\mathcal{H}|}\right\}_{s=1}^{S}$ to upper bound the projection terms [14], and by using the Schur complement to convert quadratic and conic constraints into linear inequality constraints [28], (13) can be re-stated in the following standard SDP form:

$$
\begin{aligned}
& \min _{\substack{\mathbf{V}, \mathbf{p}_{c}, \mathbf{q}_{c}, \mathbf{d} \\
\alpha, \mathbf{y} \succeq \mathbf{0}, \mathbf{u}_{s} \succeq \mathbf{0} \\
\mathbf{z} \succ \mathbf{0}}} C\left(\mathbf{V}, \mathbf{p}_{c}, \mathbf{d}\right)+c_{z} \mathbf{1}_{|\mathcal{H}|}^{\top} \mathbf{z}+c_{R} \alpha+\frac{c_{R}}{S(1-\beta)} \mathbf{1}_{S}^{\top} \mathbf{y} \\
& \text { s. to } \mathbf{V} \succeq \mathbf{0}, 13 \mathrm{~b}-13 \mathrm{e} \text {, and } \\
& {\left[\begin{array}{ccc}
z_{h} & 0 & P_{c, h} \\
0 & z_{h} & Q_{c, h} \\
P_{c, h} & Q_{c, h} & z_{h}
\end{array}\right] \succeq 0} \\
& {\left[\begin{array}{ccc}
-S_{h}^{2} & Q_{c, h} & d_{h}-P_{c, h} \\
Q_{c, h} & -1 & 0 \\
d_{h}-P_{c, h} & 0 & -1
\end{array}\right] \preceq \mathbf{0} \quad \forall h \in \mathcal{H}(14 \mathrm{c})} \\
& \mathbf{0} \preceq \mathbf{p}_{c} \preceq \mathbf{d} \\
& \mathbf{q}_{c} \preceq \tan \theta\left(\mathbf{d}-\mathbf{p}_{c}\right) \\
& -\mathbf{q}_{c} \preceq \tan \theta\left(\mathbf{d}-\mathbf{p}_{c}\right) \\
& \mathbf{1}_{|\mathcal{H}|}^{\top} \mathbf{u}_{s} \leq \alpha+y_{s}, \\
& \mathbf{p}^{\text {av }}[s]-\mathbf{d} \preceq \mathbf{u}_{s}, \\
& \forall s=1, \ldots, S \\
& \mathbf{d} \in \mathcal{D} \text {. }
\end{aligned}
$$

Remark (load uncertainty). Although this section focused on solar irradiance forecasting errors, uncertainty in active and reactive household demands can also be accounted for in the risk-aware OID framework. For example, for the active power demand, function $r\left(\mathbf{d}, \mathbf{p}^{\text {av }}\right)=\sum_{h \in \mathcal{H}}\left[P_{h}^{\text {av }}-P_{\ell, h}-\left(d_{h}-\ell_{h}\right)\right]_{+}$ can be utilized to capture surplus of net generated active power throughout the feeder, where both $P_{h}^{\text {av }}$ and $P_{\ell, h}$ are now random variables, and $\ell_{h}$ is the counterpart of $d_{h}$ for the demanded active power. Then, the risk-aware OID problem is obtained by replacing $P_{\ell, h}$ with $\ell_{h}$ in (13b). A similar procedure can be followed for uncertain reactive loads.

Remark (optimal solution). On par with [29], [30], for distribution feeders that are radial and balanced, the semidefinite relaxation (13) is exact when the following sufficient conditions are satisfied: $\mathrm{s} 1$ ) the cost function is increasing with respect to the net active power injection; s2) the voltage angle difference $\theta_{i k}$ between nodes $i$ and $k$ is such that $-\tan ^{-1}\left(b_{i k} / g_{i k}\right) \leq$ $\theta_{i k} \leq \tan ^{-1}\left(b_{i k} / g_{i k}\right)$, with $y_{i k}=g_{i k}+j b_{i k}$ the admittance of the line $(i, j) \in \mathcal{E}$; and, s3) inverters are able to absorb a "sufficient" amount of reactive power, with specific bounds quantified in [30, Thm. 1]. Condition s2) is typically satisfied in practice, since voltage angle differences are small; condition s3) can be checked by inspecting $\mathcal{F}^{\mathrm{OID}}$; and, s1) can be satisfied by appropriate tuning of the problem parameters. For unbalanced feeders as well as meshed networks, efforts for finding sufficient conditions that ensure exactness of the semidefinite relaxation are still undergoing. However, the

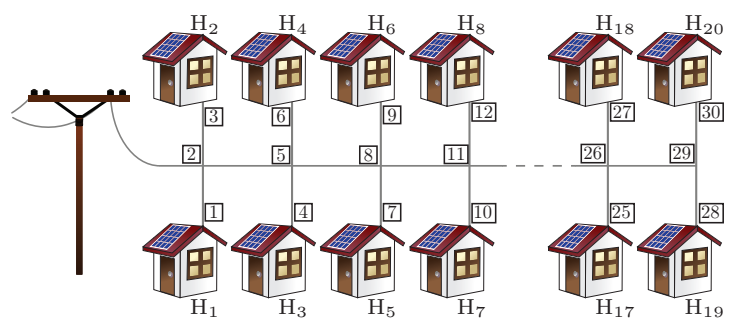

Fig. 3. Low-voltage residential network adopted for the case studies.

virtues of semidefinite relaxation have been demonstrated in e.g., [24] and [36].

Remark (uncertainty in the temperature) The formulation (13) accounts for uncertainty in the available active power through function $R_{\beta}(\alpha, \mathbf{d})$. Accordingly, it would be straightforward to translate forecasted irradiance and temperature values into forecasted power values using standard models for PV modules and inverters, see, e.g., [37], provided the probability density function of irradiance and temperature forecasting errors are available [c.f. (7) and (12)].

\section{CAse Studies}

To solve the OID problem, the distribution network operator requires: i) the Monte Carlo samples $\left\{\mathbf{p}^{\text {av }}[s] \in \mathcal{D}\right\}_{s=1}^{S}$, based on the distribution of the solar irradiation error [15]; ii) the network admittance matrix $\mathbf{Y}$; ii) the probability level $\beta$; the ratings $\left\{S_{h}\right\}$; and, v) the weighting coefficients $c_{L}, c_{P}, c_{z}, c_{R}$, which may be driven by ancillary service market strategies [5], [6] and/or security-oriented objectives. The optimization package $C V \times \sqrt{3}$ is employed to solve the OID problem in MATLAB. In all the presented tests, the rank of matrix $\mathbf{V}$ was always 1 , implying that the SDR relaxation for the power flow equations is tight.

The distribution network in Fig. 3 is considered in the test cases, which is a larger version of the fishbone system utilized in [3], [11] to assess the impact of high PV generation in residential setups. The pole-pole distance is set to $30 \mathrm{~m}$, while the lengths of the drop lines are set to $20 \mathrm{~m}$. The values of the line impedances are adopted from [3].

The 20 houses shown in Fig. 3 feature fixed roof-top PV systems, with a DC-AC derating coefficient of 0.77 . The DC ratings of the houses are as follows: $5.52 \mathrm{~kW}$ for houses $\mathrm{H}_{1}, \mathrm{H}_{3}, \mathrm{H}_{6}, \mathrm{H}_{7}, \mathrm{H}_{8}, \mathrm{H}_{9}, \mathrm{H}_{11}, \mathrm{H}_{14}, \mathrm{H}_{16}$, and $\mathrm{H}_{19}$; $8.00 \mathrm{~kW}$ for houses $\mathrm{H}_{2}, \mathrm{H}_{10}, \mathrm{H}_{12}, \mathrm{H}_{13}, \mathrm{H}_{18}, \mathrm{H}_{20}$; and, 5.70 $\mathrm{kW}$ for the remaining houses. The minimum power factor for the inverters is set to 0.85 , and it is assumed that the PV inverters are oversized by $10 \%$ of their AC rating [7]. To account for forecasting errors, the available powers are modeled as $P_{h}^{\text {av }}=\bar{P}_{h}^{\text {av }}+\Delta_{h}$, with $\bar{P}_{h}^{\text {av }}$ the (known) forecasted value and $\Delta_{h}$ the (random) forecasting error. The hourly forecasted values of the available powers $\left\{\bar{P}_{h}^{\text {av }}\right\}$ are computed using the System Advisor Mode 4 of the National Renewable Energy Laboratory, based on typical meteorological year data for Minneapolis, MN, during the month of July. Hourly PV

\footnotetext{
${ }^{3}$ [Online] http: / / cvxr. com/cvx/

${ }^{4}$ [Online] https: //sam.nrel.gov/.
} 


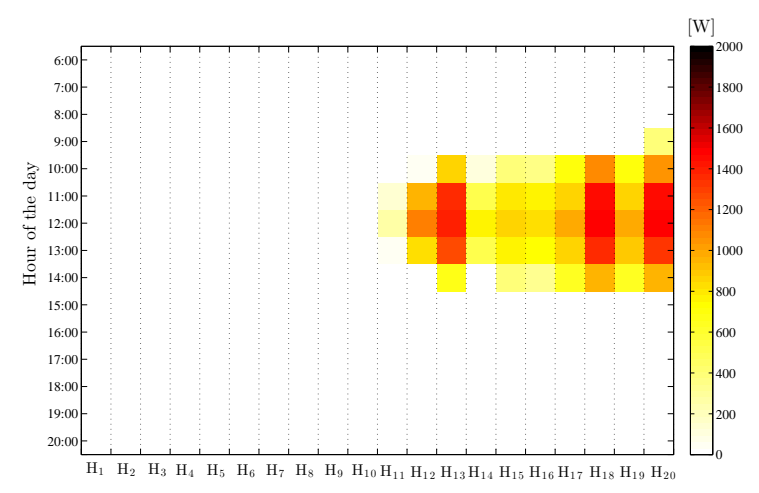

(a)

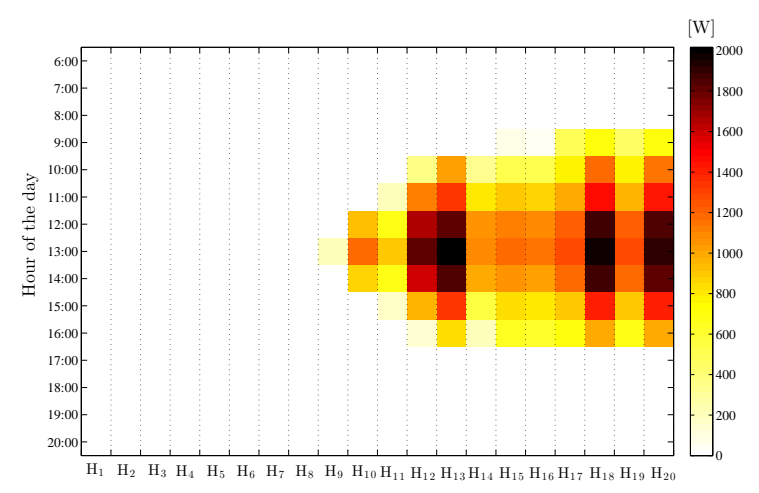

(b)

Fig. 4. Dispatched inverters: provisioned active power curtailment for (a) $c_{R}=0.01$ and (b) $c_{R}=10$.

generation in the interval $\mathcal{T}:=\{6 \mathrm{AM}, \ldots, 8 \mathrm{PM}\}$ is considered. A zero-mean truncated Gaussian distribution is adopted for $\Delta_{h}$, with truncation at the 0.3 th and 99.7 th percentiles; see e.g., [15]. Random variables $\left\{\Delta_{h}\right\}$ are correlated across houses, and an exponentially decreasing correlation function $\mathbb{E}\left\{\Delta_{h} \Delta_{h^{\prime}}\right\}=\sigma_{h} \sigma_{h^{\prime}} e^{-d\left(h, h^{\prime}\right) / \tau}$ is used, where $\sigma_{h}$ is the standard deviation of $\Delta_{h}, d\left(h, h^{\prime}\right)$ the distance between houses $h$ and $h^{\prime}$, and $\tau=300[\mathrm{~m}]$.

The residential load profile is obtained from the Open Energy Info database 5 and the "base load" experienced in downtown Saint Paul, MN, during the month of July is used for this test case. To generate different load profiles, the base active power profile is perturbed using a truncated Gaussian random variable with zero mean and standard deviation 200 $\mathrm{W}$, and a power factor of 0.9 is presumed [3]. Finally, voltages $V^{\min }$ and $V^{\max }$ are set to $0.917 \mathrm{pu}$ and $1.042 \mathrm{pu}$, respectively in this case study (see e.g., page 11 of the CAN3-C235-83 standard). The voltage at the secondary of the transformer is set at $1.02 \mathrm{pu}$, to ensure a minimum voltage magnitude of $0.917 \mathrm{pu}$ when the PV inverters do not generate power.

In the first setup, the standard deviation of the solar power prediction error $\sigma_{h}$ amounts to $10 \%$ of the forecasted value [15] (that is, $\sigma_{h} / \bar{P}_{h}^{\text {av }}=0.1$ ); $S=1000 ; \beta=0.95$; $c_{z}=0.9$ to capture fixed rewards when inverters are called upon providing ancillary services; cost $C\left(\mathbf{V}, \mathbf{p}_{c}, \mathbf{d}\right)$ is set to

${ }^{5}$ [Online] http://en.openei.org/datasets/node/961
TABLE I

PROVISIONED ANCILLARY SERVICES FOR DIFFERENT RISK LEVELS $\left(\beta=0.95, c_{L}=1, c_{P}=0.5, c_{z}=0.9\right)$

\begin{tabular}{l||c|c|c|} 
& $P_{c}^{\text {tot }}[\mathrm{kWh}]$ & $Q_{c}^{\text {tot }}[\mathrm{kVAr}]$ & $N^{\text {tot }}$ \\
\hline \hline No risk & 27.83 & 4.65 & 44 \\
$c_{R}=0.01$ & 38.60 & 8.45 & 44 \\
$c_{R}=0.1$ & 40.26 & 8.53 & 56 \\
$c_{R}=1$ & 44.05 & 9.75 & 59 \\
$c_{R}=10$ & 79.15 & 14.83 & 86
\end{tabular}

TABLE II

PROVISIONED ANCILLARY SERVICES FOR DIFFERENT PROBABILITY LEVELS $\beta\left(c_{L}=1, c_{P}=1, c_{z}=0.9, c_{R}=1\right)$

\begin{tabular}{l||c|c|c|} 
& $P_{c}^{\text {tot }}[\mathrm{kWh}]$ & $Q_{c}^{\text {tot }}[\mathrm{kVAr}]$ & $N^{\text {tot }}$ \\
\hline \hline No risk & 27.83 & 4.65 & 44 \\
$\beta=0.85$ & 41.05 & 9.51 & 55 \\
$\beta=0.90$ & 41.35 & 9.65 & 57 \\
$\beta=0.95$ & 44.05 & 9.75 & 59 \\
$\beta=0.99$ & 49.55 & 10.22 & 64
\end{tabular}

$C\left(\mathbf{V}, \mathbf{p}_{c}, \mathbf{d}\right)=c_{L}\left(\mathbf{1}_{|\mathcal{H}|}^{\top}\left(\mathbf{d}-\mathbf{p}_{c}-\mathbf{p}_{\ell}\right)+P_{0}\right)+c_{P} \mathbf{1}_{|\mathcal{H}|}^{\top} \mathbf{p}_{c}$, with $c_{L}=1$ and $c_{P}=0.5$. The amount of active power that can be curtailed by each inverter during the day is illustrated in Fig. 4 for $c_{R}=0.01$ (lower weight given to the CVaR) and $c_{R}=10$ (low CVaR objectives). It can be clearly seen that the amount of active power provisioned from each inverter increases with the increasing of $c_{R}$, thus ensuring an enhanced system protection against unexpected boosts in the solar irradiation. Clearly, the enhanced system protection comes at the expense of a higher reward for customers providing this ancillary service (modeled by the term $c_{P} \mathbf{1}_{|\mathcal{H}|}^{\top} \mathbf{p}_{c}$ ). As observed also in [11], inverters with higher ratings may be required to curtail more active power. To facilitate fairness among customers, the term $\left\|\boldsymbol{\Pi} \mathbf{p}_{c}\right\|_{2}$ can be included in 13a), where $\boldsymbol{\Pi}:=\mathbf{I}_{|\mathcal{H}|}-\frac{1}{|\mathcal{H}|} \mathbf{1}_{|\mathcal{H}| \times 1} \mathbf{1}_{|\mathcal{H}| \times 1}^{\top}$. Finally, notice that for $c_{R}=10$, an increased number of inverters are required to curtail active power.

This trend is confirmed by the results reported in Table \ where: $P_{c}^{\text {tot }}:=\sum_{t \in \mathcal{T}} \sum_{h \in \mathcal{H}} P_{c, h}(t)$ denotes the conglomerate active power curtailment that is procured during the day, with $P_{c, h}(t)$ the amount of power that can be curtailed from inverter $h$ at time $t ; Q_{c}^{\text {tot }}:=\sum_{t \in \mathcal{T}} \sum_{h \in \mathcal{H}}\left|Q_{c, h}(t)\right|$ the overall reactive power procured for reactive support purposes; and $N^{\text {tot }}$ the total number of inverters called upon providing ancillary services over $\mathcal{T}$ (out of $20 \times|\mathcal{T}|=360$ ). These values are compared with the "no risk" setup, where the solar forecasting errors are neglected, and ancillary services are provisioned by solving (3) with $\left\{P_{h}^{\text {av }}\right\}$ replaced by $\left\{\bar{P}_{h}^{\text {av }}\right\}$. Clearly, considering only the expected available powers $\left\{\bar{P}_{h}^{\text {av }}\right\}$ yields an underestimate of the amount of active and reactive power reserves that may be needed to ensure voltage regulation. This corroborates the ability of the proposed approach to trade off risks of overvoltage conditions for the amount of ancillary services to be secured [5].

Table quantifies the procured active and reactive reserves for different values for the probability level $\beta$. In this setup, the other problem parameters are set as $c_{L}=1, c_{P}=1, c_{z}=0.9$ and $c_{R}=1$. With the increasing of $\beta$, progressively higher 
TABLE III

PROVISIONED ANCILLARY SERVICES FOR DIFFERENT UNCERTAINTY $\operatorname{LEVELS}\left(c_{L}=1, c_{P}=1, c_{z}=0.9, c_{R}=1, \beta=0.95\right)$

\begin{tabular}{l||c|c|c|}
$\sigma_{h} / \bar{P}_{h}^{\mathrm{av}}$ & $P_{c}^{\text {tot }}[\mathrm{kWh}]$ & $Q_{c}^{\text {tot }}[\mathrm{kVAr}]$ & $N^{\text {tot }}$ \\
\hline \hline 0 & 27.83 & 4.65 & 44 \\
0.05 & 42.12 & 9.19 & 57 \\
0.10 & 44.05 & 9.75 & 59 \\
0.15 & 46.25 & 10.35 & 63 \\
0.20 & 51.93 & 11.64 & 65
\end{tabular}

solar irradiation conditions are considered in the risk-aware OID problem [cf. (5)]; this explains why the amount of active and reactive powers reserves that are secured by the OID framework increases. A similar trend can be noticed in Table III] where values for the standard deviation of the forecasting errors are tested. Notice that the results for $\sigma_{h}=0$ (i.e., perfect knowledge of the solar irradiation conditions) coincide with the "no risk" setup of Tables [ and III As expected, the higher is $\sigma_{h}$, the higher is the number of inverters that may be required to provide ancillary services.

\section{COncluding Remarks}

The present paper dealt with ancillary service provisioning in distribution systems in the presence of solar irradiance forecasting errors. The proposed uncertainty-aware OID identifies the subset of critical inverters that strongly impact both voltage profile and network performance objectives, and quantifies the amount of active and reactive powers to be procured from each inverter. The CVaR measure was utilized to capture (and minimize) the risk of overvoltages throughout the feeder. Although the formulated OID task involves the solution of a nonconvex mixed-integer nonlinear program, a convex relaxation was developed by leveraging sparsity-promoting regularization approaches and semidefinite relaxation techniques. Using realworld PV-generation and load-profile data, it is shown how the proposed framework can trade of the risk of PV generation surplus for the amount of ancillary services to be provisioned.

\section{APPENDIX}

The dependence between active power injections and voltage magnitudes in low-voltage distribution systems is briefly analyzed in the following.

Consider a low-voltage single-phase distribution line, and let $Z:=R+j \omega L$ be its impedance, where $R, L$, and $\omega$ denote the per-unit-length line resistance and inductance, and electrical frequency. Typical values for $R$ and $L$ are on the order of $10^{-1} \Omega / \mathrm{km}$ and $10^{-4} \mathrm{H} / \mathrm{km}$, respectively (see e.g., the specifications of cables NS 90 3/0 AWG utilized for the pole-to-pole connections and cables NS 90 1/0 AWG for drop lines [3]). Let $Y:=1 / Z=, G:=\Re\{Y\}=R /|Z|^{2}$ and $B:=\Im\{Y\}=-\omega L /|Z|^{2}$. Further, let $\left|V_{1}\right| e^{j \theta_{1}}$ and $\left|V_{2}\right| e^{j \theta_{2}}$ denote the phasors for the voltages at the two end points of the line. Although a single line is considered for simplicity, claims naturally extend to low-voltage systems with arbitrary topologies.
The active- and reactive-power injections at node 1 of the line are given by

$$
\begin{aligned}
& P_{1}=\left|V_{1}\right|^{2} G-\left|V_{1}\right|\left|V_{2}\right| G \cos (\theta)-\left|V_{1}\right|\left|V_{2}\right| B \sin (\theta), \\
& Q_{1}=-\left|V_{1}\right|^{2} B+\left|V_{1}\right|\left|V_{2}\right| B \cos (\theta)-\left|V_{1}\right|\left|V_{2}\right| G \cos (\theta),
\end{aligned}
$$

where $\theta:=\theta_{1}-\theta_{2}$. Similarly, the active- and reactive-power injections at node 2 of the line are given by

$$
\begin{aligned}
& P_{2}=\left|V_{2}\right|^{2} G-\left|V_{1}\right|\left|V_{2}\right| G \cos (\theta)+\left|V_{1}\right|\left|V_{2}\right| B \sin (\theta), \\
& Q_{2}=-\left|V_{2}\right|^{2} B+\left|V_{1}\right|\left|V_{2}\right| B \cos (\theta)+\left|V_{1}\right|\left|V_{2}\right| G \cos (\theta) .
\end{aligned}
$$

With regard to $15 \mathrm{a}-116 \mathrm{~b}$, define the sensitivity matrix

$$
\mathbf{S}\left(\left|V_{1}\right|,\left|V_{2}\right|, \theta_{1}, \theta_{2}\right):=\left[\begin{array}{cccc}
\frac{\partial P_{1}}{\partial\left|V_{1}\right|} & \frac{\partial P_{1}}{\partial V_{2} \mid} & \frac{\partial P_{1}}{\partial \theta_{1}} & \frac{\partial P_{1}}{\partial \theta_{2}} \\
\frac{\partial P_{2}}{\partial\left|V_{1}\right|} & \frac{\partial P_{2}}{\partial V_{2}} & \frac{\partial P_{2}}{\partial \theta_{1}} & \frac{\partial P_{2}}{\partial \theta_{2}} \\
\frac{\partial Q_{1}}{\partial\left|V_{1}\right|} & \frac{\partial Q_{1}}{\partial\left|V_{2}\right|} & \frac{\partial Q_{1}}{\partial \theta_{1}} & \frac{\partial Q_{1}}{\partial \theta_{2}} \\
\frac{\partial Q_{2}}{\partial\left|V_{1}\right|} & \frac{\partial Q_{2}}{\partial\left|V_{2}\right|} & \frac{\partial Q_{2}}{\partial \theta_{1}} & \frac{\partial Q_{2}}{\partial \theta_{2}}
\end{array}\right],
$$

which relates power variations with perturbations of the voltage phasors around a given operational point.

Next, assume small voltage angle variations; that is, $\theta \ll 1$, $\cos (\theta) \approx 1$ and $\sin (\theta) \approx \theta$. Under these assumptions, we can relate sensitivities of voltage magnitudes and angles to real and reactive power injections through (17). Furthermore, since $\frac{\omega L}{R} \ll 1$ in low-voltage distribution systems (this condition is not necessarily true in medium-voltage networks), it follows that $B \ll 1$, and thus the effects of voltage magnitude and phase variations on the complex powers approximately decouples as

$$
\begin{gathered}
{\left[\begin{array}{c}
\Delta P_{1} \\
\Delta P_{2}
\end{array}\right] \approx\left[\begin{array}{cc}
2 G\left|V_{1}\right|-G\left|V_{2}\right| & -G\left|V_{1}\right| \\
-G\left|V_{2}\right| & -G\left|V_{1}\right|+2 G\left|V_{2}\right|
\end{array}\right]\left[\begin{array}{l}
\Delta\left|V_{1}\right| \\
\Delta\left|V_{2}\right|
\end{array}\right],} \\
{\left[\begin{array}{c}
\Delta Q_{1} \\
\Delta Q_{2}
\end{array}\right] \approx\left[\begin{array}{cc}
-G\left|V_{1}\right|\left|V_{2}\right| & G\left|V_{1}\right|\left|V_{2}\right| \\
G\left|V_{1}\right|\left|V_{2}\right| & -G\left|V_{1}\right|\left|V_{2}\right|
\end{array}\right]\left[\begin{array}{c}
\Delta \theta_{1} \\
\Delta \theta_{2}
\end{array}\right] .}
\end{gathered}
$$

Thus, due to the high resistance-to-inductance ratio in lowvoltage distribution lines, voltage magnitudes are more sensitive to variations in the active power flows. It follows that curtailing active power during peak generation hours represents a viable way to avoid overvoltage conditions throughout the feeder. Furthermore, the higher is the solar irradiation, the higher is the overall amount of active power that should be curtailed in order to enforce voltage regulation.

\section{REFERENCES}

[1] E. Liu and J. Bebic, "Distribution system voltage performance analysis for high-penetration photovoltaics," Feb. 2008, NREL Technical Monitor: B. Kroposki. Subcontract Report NREL/SR-581-42298.

[2] California Public Utilities Commission, "Advanced inverter technologies report," Jan. 2013, [Online] http: / / www. cpuc.ca.gov.

[3] R. Tonkoski, L. A. C. Lopes, and T. H. M. El-Fouly, "Coordinated active power curtailment of grid connected PV inverters for overvoltage prevention," IEEE Trans. on Sust. Energy, vol. 2, no. 2, pp. 139-147, Apr. 2011

[4] A. F. Vizoso, L. Piegari, and P. Tricoli, "A photovoltaic power unit providing ancillary services for smart distribution networks," in Intl. Conf. on Renewable Energies and Power Quality, Las Palmas, Spain, 2010.

[5] E. Ntakou and M. C. Caramanis, "Price discovery in dynamic power markets with low-voltage distribution-network participants," in IEEE PES Trans. \& Distr. Conf., Chicago, IL, 2014.

[6] D. Craciun and D. Geibel, "Evaluation of ancillary services provision capabilities from distributed energy supply," in Intl. Conf. on Electricity Distribution CIRED, Stockholm, June 2012. 


$$
\left[\begin{array}{c}
\Delta P_{1} \\
\Delta P_{2} \\
\Delta Q_{1} \\
\Delta Q_{2}
\end{array}\right]=\left[\begin{array}{cccc}
2 G\left|V_{1}\right|-G\left|V_{2}\right| & -G\left|V_{1}\right| & -B\left|V_{1}\right|\left|V_{2}\right| & B\left|V_{1}\right|\left|V_{2}\right| \\
-G\left|V_{2}\right| & -G\left|V_{1}\right|+2 G\left|V_{2}\right| & B\left|V_{1}\right|\left|V_{2}\right| & -B\left|V_{1}\right|\left|V_{2}\right| \\
-2 B\left|V_{1}\right|+B\left|V_{2}\right| & B\left|V_{1}\right| & -G\left|V_{1}\right|\left|V_{2}\right| & G\left|V_{1}\right|\left|V_{2}\right| \\
B\left|V_{2}\right| & B\left|V_{1}\right|-2 B\left|V_{2}\right| & G\left|V_{1}\right|\left|V_{2}\right| & -B\left|V_{1}\right|\left|V_{2}\right|
\end{array}\right]\left[\begin{array}{c}
\Delta\left|V_{1}\right| \\
\Delta\left|V_{2}\right| \\
\Delta \theta_{1} \\
\Delta \theta_{2}
\end{array}\right] .
$$

[7] K. Turitsyn, P. Sulc, S. Backhaus, and M. Chertkov, "Options for control of reactive power by distributed photovoltaic generators," Proc. of the IEEE, vol. 99, no. 6, pp. 1063-1073, 2011.

[8] P. Jahangiri and D. C. Aliprantis, "Distributed Volt/VAr control by PV inverters," IEEE Trans. Power Syst., vol. 28, no. 3, pp. 3429-3439, Aug. 2013.

[9] S. Bolognani and S. Zampieri, "A distributed control strategy for reactive power compensation in smart microgrids," IEEE Trans. on Autom. Control, vol. 58, no. 11, pp. 2818-2833, 2013.

[10] A. Samadi, R. Eriksson, L. Soder, B. G. Rawn, and J. C. Boemer, "Coordinated active power-dependent voltage regulation in distribution grids with pv systems," IEEE Trans. on Power Del., vol. 29, no. 3, pp. 1454-1464, June 2014.

[11] E. Dall'Anese, S. V. Dhople, and G. B. Giannakis, "Optimal dispatch of photovoltaic inverters in residential distribution systems," IEEE Trans. Sustainable Energy, vol. 5, no. 2, pp. 487-497, Apr. 2014.

[12] E. Dall'Anese, S. V. Dhople, B. B. Johnson, and G. B. Giannakis, "Decentralized optimal dispatch of photovoltaic inverters in residential distribution systems," IEEE Trans. on Energy Conversion, 2014, to appear. Also available at: http://arxiv.org/abs/1403.1341.

[13] A. McNeil, R. Frey, and P. Embrechts, Quantitative Risk Management: Concepts Techniques and Tools. Princeton University Press, 2005.

[14] R. T. Rockafellar and S. Uryasev, "Optimization of conditional valueat-risk," Journal of Risk, vol. 2, no. 3, pp. 21-41, 2000.

[15] P. Bacher, H. Madsen, and H. A. Nielsen, "Online short-term solar power forecasting," Solar Energy, vol. 83, no. 10, pp. 1772-1783, 2009.

[16] E. Lorenz, J. Hurka, D. Heinemann, and H. G. Beyer, "Irradiance forecasting for the power prediction of grid-connected photovoltaic systems," IEEE J. Sel. Top. App. Earth Observ. and Rem. Sensing, vol. 2, no. 1, pp. 2-10, 2009.

[17] E. Sjodin, D. F. Gayme, and U. Topcu, "Risk-mitigated optimal power flow for wind powered grids," in American Control Conf., Montreal, Canada, June 2012.

[18] J. Warrington, P. Goulart, S. Mariethoz, and M. Morari, "Policy-based reserves for power systems," IEEE Trans. Power Syst., vol. 28, no. 4 , pp. 4427-4437, Nov 2013.

[19] T. Summers, J. Warrington, M. Morari, and J. Lygeros, "Stochastic optimal power flow based on convex approximations of chance constraints," in Power Systems Computation Conf., Wroclaw, Poland, Aug. 2014.

[20] Q. Wang, Y. Guan, and J. Wang, "A chance-constrained two-stage stochastic program for unit commitment with uncertain wind power output," IEEE Trans. Power Syst., vol. 27, no. 1, pp. 206-215, Feb 2012.

[21] D. Bienstock, M. Chertkov, and S. Harnett, "Chance constrained optimal power flow: Risk-aware network control under uncertainty," 2013, [Online] http://arxiv.org/abs/1209.5779.

[22] M. Kloppel, A. Gabash, A. Geletu, and P. Li, "Chance constrained optimal power flow with non-gaussian distributed uncertain wind power generation," in Intl. Conf. on Environment and Electrical Engineering, May 2013, pp. 265-270.

[23] Y. Zhang and G. B. Giannakis, "Robust optimal power flow with wind integration using conditional value-at-risk," in 4th Intl. Conf. on Smart Grid Communications, Vancouver, Canada, Oct. 2013.

[24] E. Dall'Anese, H. Zhu, and G. B. Giannakis, "Distributed optimal power flow for smart microgrids," IEEE Trans. Smart Grid, vol. 4, no. 3, pp. 1464-1475, Sep. 2013.

[25] J. Lavaei and S. H. Low, "Zero duality gap in optimal power flow problem," IEEE Trans. Power Syst., vol. 1, no. 1, pp. 92-107, 2012.

[26] W. H. Kersting, Distribution System Modeling and Analysis. 2nd ed., Boca Raton, FL: CRC Press, 2007.

[27] A. T. Puig, A. Wiesel, G. Fleury, and A. O. Hero, "Multidimensional shrinkage-thresholding operator and group LASSO penalties," IEEE Sig. Proc. Letters, vol. 18, no. 6, pp. 363-366, Jun. 2011.

[28] L. Vandenberghe and S. Boyd, "Semidefinite programming," SIAM Review, vol. 38, no. 1, pp. 49-95, Mar. 1996.

[29] J. Lavaei, D. Tse, and B. Zhang, "Geometry of power flows and optimization in distribution networks," IEEE Trans. Power Syst., vol. 29, no. 2, pp. 572-583, March 2014.

[30] B. Zhang, A. Y. Lam, A. Dominguez-Garcia, and D. Tse, "An optimal and distributed method for voltage regulation in power distribution systems," IEEE Trans. Power Syst., to appear. See also: http://arxiv.org/abs/1204.5226.

[31] A. Nesterov and Y. Nemirovski, Interior-Point Polynomial Algorithms in Convex Programming. SIAM Studies in Applied Mathematics, 1994.

[32] R. A. Jabr, "Exploiting sparsity in SDP relaxations of the OPF problem," IEEE Trans. Power Syst., vol. 2, no. 27, pp. 1138-1139, May 2012.

[33] F. Chassin, E. Mayhorn, M. Elizondo, and S. Lus, "Load modeling and calibration techniques for power system studiess," in North American Power Symp., Boston, MA, Aug. 2011.

[34] D. K. Molzahn, B. C. Lesieutre, and C. L. DeMarco, "Approximate representation of ZIP loads in a semidefinite relaxation of the OPF problem," IEEE Trans. Power Syst., Letters, vol. 29, no. 4, pp. 18641865, July 2014.

[35] S. Boyd and L. Vandenberghe, Convex Optimization. Cambridge University Press, 2004.

[36] L. Gan and S. H. Low, "Convex relaxations and linear approximation for optimal power flow in multiphase radial networks," 2014, [Online] http://arxiv.org/abs/1406.3054.

[37] G. Masters, Renewable and Efficient Electric Power Systems. Wiley, 2004. 